\title{
Chemical abundances in Seyfert galaxies - V. The discovery of shocked emission outside the AGN ionization axis
}

\author{
R. A. Riffel, ${ }^{1 \star}$ O. L. Dors, ${ }^{2}$ M. Armah, ${ }^{2}$ T. Storchi-Bergmann, ${ }^{3}$, A. Feltre, ${ }^{4}$ \\ G. F. Hägele, ${ }^{5,6}$ M. V. Cardaci, ${ }^{5,6}$ D. Ruschel-Dutra, ${ }^{7}$ A. C Krabbe, ${ }^{2}$ E. Pérez-Montero, ${ }^{8}$ \\ N. L. Zakamska, ${ }^{9}$ I. C. Freitas ${ }^{10}$ \\ ${ }^{1}$ Departamento de Física, Centro de Ciências Naturais e Exatas, Universidade Federal de Santa Maria, 97105-900, Santa Maria, RS, Brazil \\ ${ }^{2}$ Universidade do Vale do Paraíba, Av. Shishima Hifumi, 2911, Cep 12244-000, São José dos Campos, SP, Brazil \\ ${ }^{3}$ Instituto de Física, Universidade Federal do Rio Grande do Sul, CP 15051, Porto Alegre, RS, 91501-970, Brazil \\ ${ }^{4}$ INAF - Osservatorio di Astrofisica e Scienza dello Spazio di Bologna, Via P. Gobetti 93/3, 40129 Bologna, Italy \\ ${ }^{5}$ Instituto de Astrofísica de La Plata (CONICET-UNLP), Argentina \\ ${ }^{6}$ Facultad de Ciencias Astronómicas y Geofísicas, Universidad Nacional de La Plata, Paseo del Bosque s/n, 1900 La Plata, Argentina \\ ${ }^{7}$ Departamento de Física, Universidade Federal de Santa Catarina, P.O. Box 476, 88040-900, Florianópolis, SC, Brazil \\ ${ }^{8}$ Instituto de Astrofísica de Andalucía, Camino Bajo de Huétor s/n, Aptdo. 3004, E18080-Granada, Spain. \\ ${ }^{9}$ Department of Physics \& Astronomy, Johns Hopkins University, Bloomberg Center, 3400 N. Charles St, Baltimore, MD 21218, USA \\ ${ }^{10}$ Colégio Politécnico, Universidade Federal de Santa Maria, Santa Maria, 97105-900 RS, Brazil
}

Accepted XXX. Received YYY; in original form ZZZ

\begin{abstract}
We present maps for the electron temperature in the inner kpc of three luminous Seyfert galaxies: Mrk 79, Mrk 348, and Mrk 607 obtained from Gemini GMOS-IFU observations at spatial resolutions of $\sim 110-280 \mathrm{pc}$. We study the distributions of electron temperature in active galaxies and find temperatures varying in the range from $\sim 8000$ to $\gtrsim 30000 \mathrm{~K}$. Shocks due to gas outflows play an important role in the observed temperature distributions of Mrk 79 and Mrk 348, while standard photoionization models reproduce the derived temperature values for Mrk 607. In Mrk 79 and Mrk 348, we find direct evidence for shock-ionization with overall orientation orthogonal to the ionization axis, where shocks can be easily observed as the AGN radiation field is shielded by the nuclear dusty torus. This also indicates that even when the ionization cones are narrow, the shocks can be much wider-angle.
\end{abstract}

Key words: galaxies: Seyfert - galaxies: active - galaxies: abundances - galaxies: ISM

\section{INTRODUCTION}

Active Galactic Nuclei (AGN) present in their spectra strong emission lines whose relative intensities can be used to estimate or characterize the physical and chemical properties of the gas phase in these objects, such as the nature and strength of the interstellar radiation fields, chemical abundance, local temperature, and gas density. The high luminosity of the AGN continuum and these emission lines have made AGN essential in the studies of chemical evolution of galaxies across the Hubble time. Photoionization models of the Narrow Line Region (NLR) have often been used for determining chemical abundances of heavy elements in AGN host galaxies. The pioneering work by Ferland \& Netzer (1983), who used the first version of the photoionization Cloudy code, showed that NLR exhibit metallicities in the range $0.1<\left(Z / Z_{\odot}\right)<1.0$. Thereafter, several studies exploiting photoionization models have been carried out aiming to estimate the metallicity of AGN host

\footnotetext{
^ E-mail: rogemar@ufsm.br
}

galaxies in the local universe (e.g. Storchi-Bergmann et al. 1998; Feltre et al. 2016; Castro et al. 2017) and at high redshifts (e.g. Dors et al. 2018; Mignoli et al. 2019; Guo et al. 2020).

In spite of the wide use of photoionization models in the estimation of gas metallicities, their application in deriving elemental abundances has several limitations. Firstly, it is necessary to assume an incident Spectral Energy Distribution as one of the input parameters of the models, which is generally represented by the power law component of the non-thermal X-ray radiation with continuum between $2 \mathrm{keV}$ and $2500 \AA$ with a spectral index $\alpha_{\text {ox }}$ (Marchese et al. 2012; Dors et al. 2019). Detailed photoionization models used to derive $\mathrm{O}$ and $\mathrm{N}$ abundances (Dors et al. 2017) and bayesian-like approach (Pérez-Montero et al. 2019) have predicted $\alpha_{o x}$ to be higher than -1.2 for Seyfert 2 nuclei. Conversely, measurements of $\alpha_{o x}$ by Miller et al. (2011) indicate that most AGN have $\alpha_{o x}$ of the order of -1.4 and even lower values $(\sim-2.0)$ can be derived for these objects. This indicates that an extra physical process is missing in the models (probably shocks, e.g., Contini 2019). Additionally, the use of emission line intensities observed in a limited spectral range 

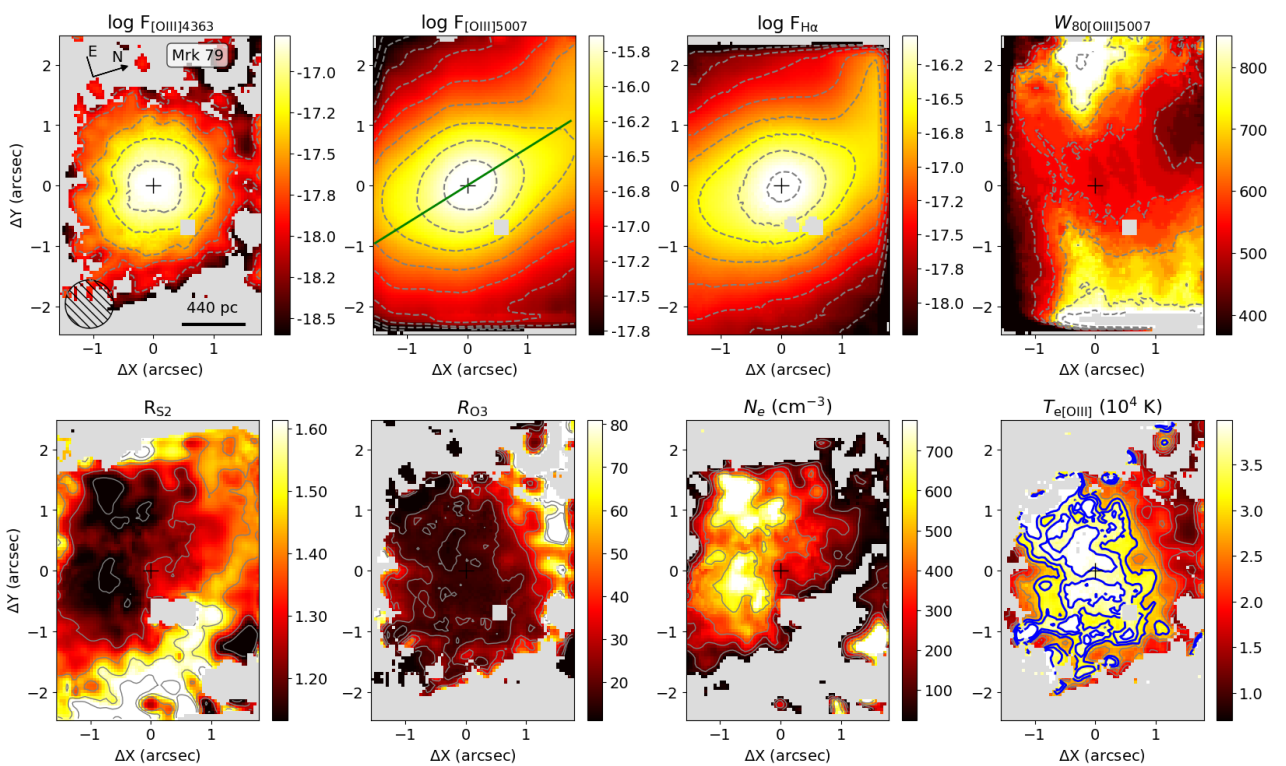

Figure 1. Maps for Mrk 79, which has a bolometric luminosity of $\log L_{\mathrm{bol}} /\left(\mathrm{erg} \mathrm{s}^{-1}\right)=45.0$ and a distance of $d=91.6 \mathrm{Mpc}$ (see Freitas et al. 2018). Top row: Emission-line flux distributions and $W_{80}$ map for the [O III]5007 emission line. Bottom row: flux line ratios, $N_{e}$ and $T_{\mathrm{e}}[\mathrm{OIII}]$ maps. The central crosses mark the position of the continuum peak, the circle shows the seeing disc, the spatial scale and orientation are shown in the [O III] $\lambda 4363$ flux map. Gray regions correspond to locations where the emission-lines were not detected at with a $\mathrm{S} / \mathrm{N}>3$. The contours show the levels of each map. Blue contours in the $T_{\mathrm{e}}[\mathrm{OIII}]$ map correspond to values larger than $30000 \mathrm{~K}$, where the uncertainties using $R_{\mathrm{O} 3}$ to derive $T_{\mathrm{e} \text { [OIII] }}$ are large. The green line on the [O III] flux maps shows the orientation of the AGN ionization axis, as obtained from HST images by Schmitt et al. (2003).

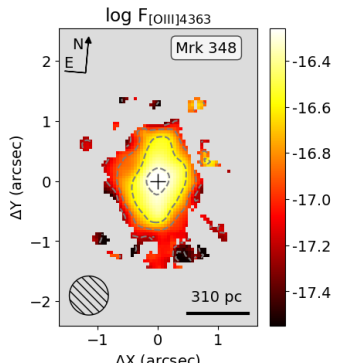

$\mathrm{R}_{\mathrm{S} 2}$

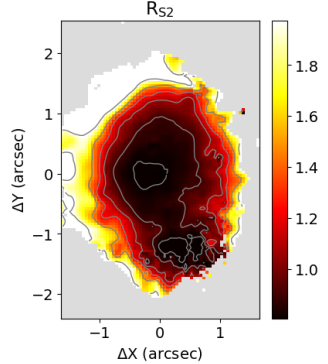

$\log \mathrm{F}_{[0 \mathrm{III}] 5007}$

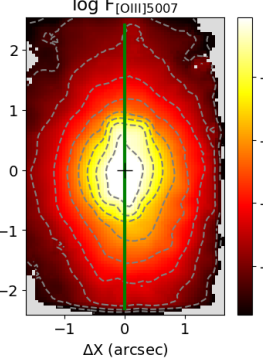

$R_{03}$

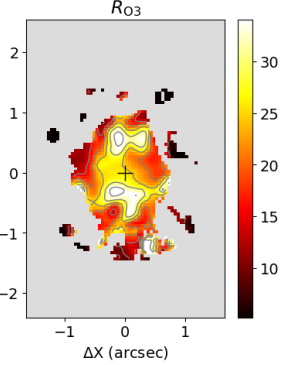

$\log \mathrm{F}_{[\mathrm{NIII}} \mathrm{f755}$

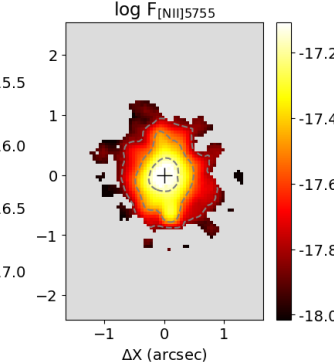

$R_{\mathrm{N} 2}$

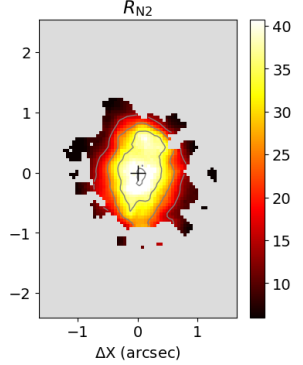

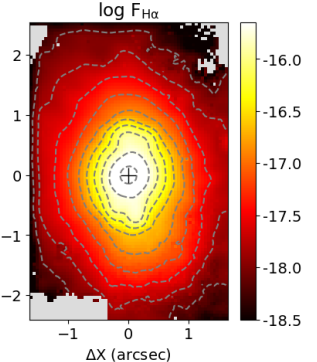

$W_{80[01115007}$

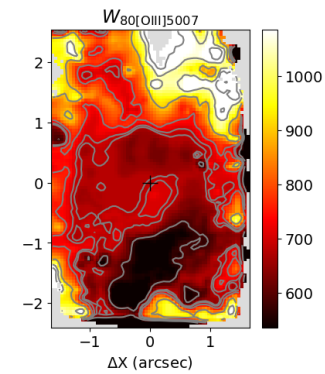

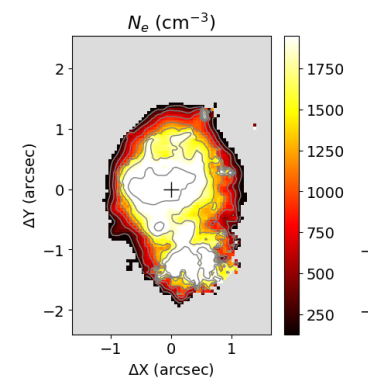
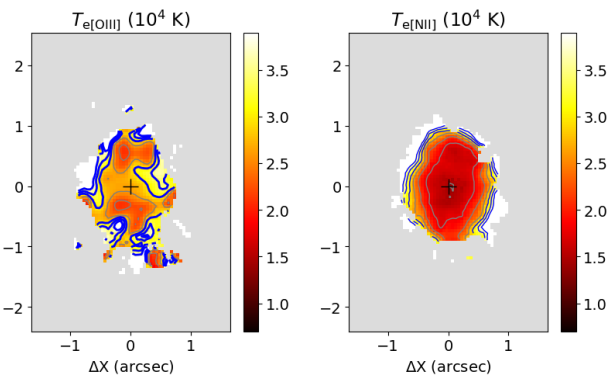

Figure 2. Maps for Mrk $348\left(\log L_{\mathrm{bol}} /\left(\mathrm{erg} \mathrm{s}^{-1}\right)=45.3, d=63.9 \mathrm{Mpc}\right)$. Emission-line flux distributions (top row), flux line ratios and $W_{80[\mathrm{OII}]}$ (middle row), and $N_{e}, T_{\mathrm{e}[\mathrm{OIII}]}$ and $T_{\mathrm{e}[\mathrm{NII}]}$ maps (bottom row). The labels are the same as for Fig. 1. 

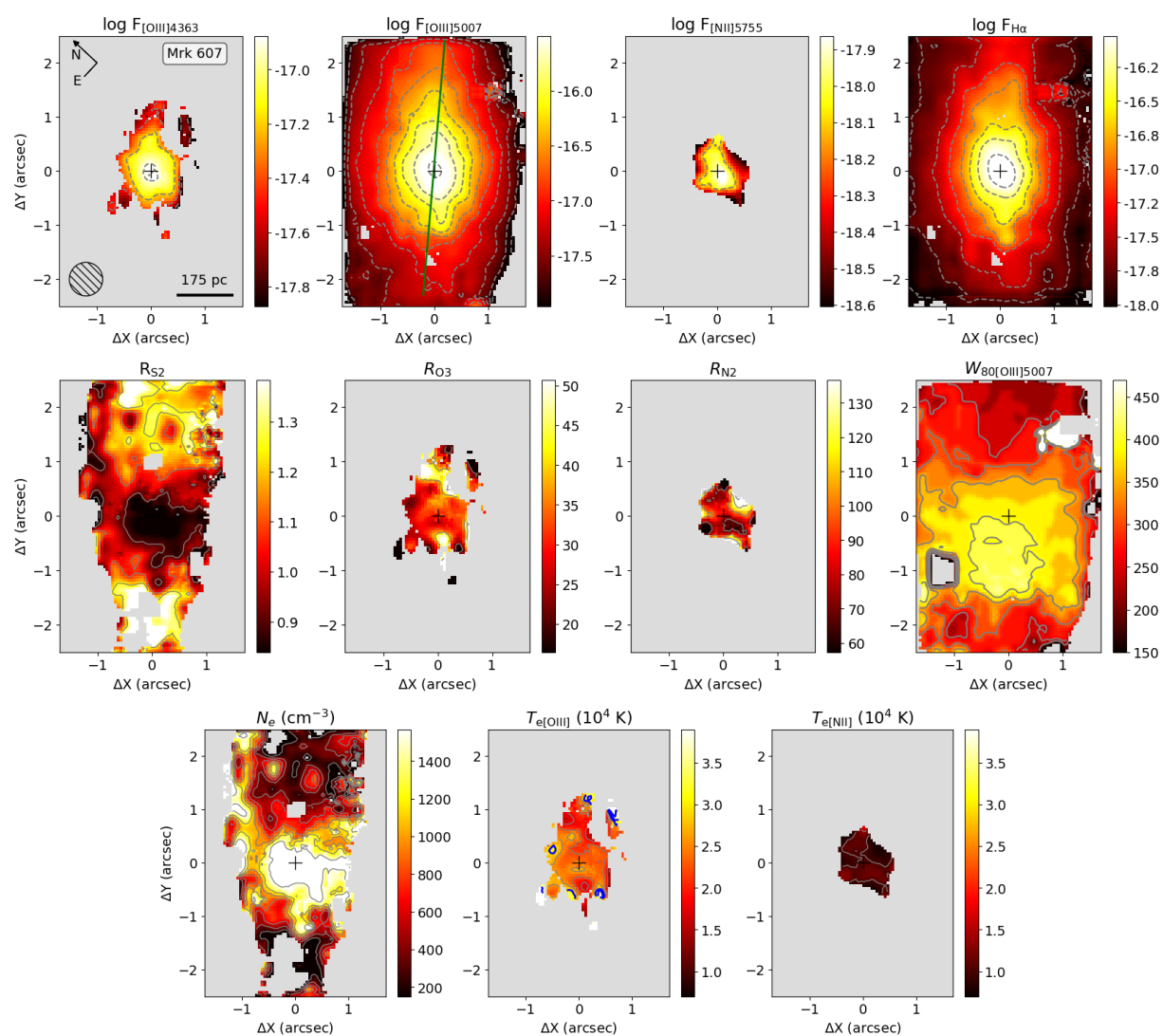

Figure 3. Same as Fig. 2 but for Mrk $607\left(\log L_{\mathrm{bol}} /\left(\mathrm{erg} \mathrm{s}^{-1}\right)=43.4, d=36.1 \mathrm{Mpc}\right)$.

can produce a degeneracy in the models (e.g. Davies et al. 2014) and, consequently, uncertainties in the chemical abundance values.

Direct determination of abundances, known as the $T_{\mathrm{e}}$-method which is based on the observational determination of the electron temperature $\left(T_{\mathrm{e}}\right)$, yields more reliable abundance estimations because it circumvents the aforementioned problems of photoionization models. Recently, Dors et al. (2020) presented a new methodology for the $T_{\mathrm{e}}$-method to be applied to NLRs of Seyfert 2 nuclei, introducing a new relation between temperatures of the low $\left(t_{2}\right)$ and high $\left(t_{3}\right)$ ionization gas zones derived from photoionization models. This method produces $\mathrm{O} / \mathrm{H}$ abundances slightly lower (about $0.2 \mathrm{dex}$ ) than those derived from detailed photoionization models. However, previous studies have questioned the use of the $T_{\mathrm{e}}$-method in AGN. For example, Stasińska (1984) argued that in the NLR the intensity of the $\left[\mathrm{O}_{\mathrm{III}}\right](\lambda 4949+\lambda 5007) / \lambda 4363$ line ratio, used to derive $t_{3}$ (e.g. Hägele et al. 2008), is enhanced by emissions from clouds with high gas density $\left(N_{\mathrm{e}}>10^{5} \mathrm{~cm}^{-3}\right)$, which precludes any direct determination of abundances based on the $T_{\mathrm{e}}$-method. Nagao et al. (2001) found evidence that the [O $\mathrm{III}] \lambda 4363$ line is emitted in denser $\left(N_{\mathrm{e}} \sim 10^{5-7} \mathrm{~cm}^{-3}\right)$ and obscured gas regions than those emitting $\left[\mathrm{O}_{\mathrm{III}}\right] \lambda 5007$

Shocks from AGN outflows may also affect the ionization structure and $T_{\mathrm{e}}$ distribution, particularly in regions where the AGN ionizing photons are shielded by nuclear obscuration (Contini \& Viegas 2001; Zakamska \& Greene 2014), where the contribution of photoionization to gas ionization is expected to be lower. Spatially resolved measurements of electron temperature and density are important to constrain the AGN contribution to gas ionization and to infer the gas physical conditions. Observational data that enable measurements of weak auroral lines, from which $T_{\mathrm{e}}$ can be estimated, and spatially resolved studies of $T_{\mathrm{e}}$ in AGN are seldom found in the literature (but see Revalski et al. 2018; Dahmer-Hahn et al. 2019; D'Agostino et al. 2019).

In this Letter, we present two-dimensional (2D) maps of electron temperature and electron density in the NLR, in the case in one Seyfert 1 (Mrk 79) and two Seyfert 2 (Mrk 348 and Mrk 607) galaxies, obtained from integral field spectroscopy. A thorough analysis of $T_{\mathrm{e}}$ and $N_{\mathrm{e}}$ could be performed with the use of 2D spectroscopy at spatial resolutions of a few hundred parsecs in these 3 AGN hosts. In Sect. 2, we describe the data and techniques to derive $T_{\mathrm{e}}$ and $N_{\mathrm{e}}$. In Sect. 3 we present and discuss the results, while the conclusions are given in Sect. 4.

\section{METHODOLOGY}

We use the Gemini Multi-Object Spectrograph (GMOS, AllingtonSmith et al. 2002) Integral Field Unit (IFU) data to map the $T_{\mathrm{e}}$ and $N_{\mathrm{e}}$ in the inner few hundred parsecs of the nearby luminous Seyfert galaxies Mrk 79 (Sy 1, SBb), Mrk 348 (Sy 2, SA(s)0/a) and Mrk 607 (Sy 2, Sa). These galaxies were selected from the sample of Freitas et al. (2018) because they have [O III] $\lambda 4363$ extended emission. The GMOS data covers the spectral range from 4300 to $7100 \AA$ with a velocity resolution of $\sim 90 \mathrm{~km} \mathrm{~s}^{-1}$ (FWHM) and spatial resolutions of $280 \pm 30$ (Mrk 79), 190 \pm 25 (Mrk 348), and $110 \pm 14 \mathrm{pc}$ (Mrk 607). The data reduction followed the standard procedures using the GEMINI.IRAF package as described by Freitas et al. (2018). We use the IFSCUBE python package (Ruschel-Dutra 2020) to fit the emission-line profiles and obtain the emissionline flux distributions. We allow the fit of up to three Gaussian 
components per emission line and the line fluxes are obtained by the sum of the fluxes of the individual components (see Suppl. Mat.). We derive the electron temperature using two sets of auroral/nebular line intensity ratios: $R_{\mathrm{O} 3}=([\mathrm{O} \mathrm{III}] \lambda \lambda 4959,5007 / \lambda 4363)$ and $R_{N 2}$ $=\left(\left[\mathrm{N}_{\text {II }}\right] \lambda \lambda 6548,6584 / \lambda 5755\right)$, using $($ Hägele et al. 2008):

$$
\frac{T_{\mathrm{e}[\mathrm{OIII}]}}{10^{4} \mathrm{~K}}=0.8254-0.0002415 R_{\mathrm{O} 3}+\frac{47.77}{R_{\mathrm{O} 3}}
$$

and

$$
\frac{T_{\mathrm{e}[\mathrm{NII}]}}{10^{4} \mathrm{~K}}=0.537+0.000253 \times R_{\mathrm{N} 2}+\frac{42.13}{R_{\mathrm{N} 2}} .
$$

The $\left[\mathrm{N}_{\mathrm{II}}\right] \lambda 5755$ emission line is not detected for Mrk 79 and, thus we are unable to calculate the $T_{\mathrm{e} \text { [NII] }}$ for this galaxy.

We estimate the electron density $\left(N_{\mathrm{e}}\right)$ from the $R_{S 2}=$ $\left[\mathrm{S}_{\text {II }}\right] \lambda 6716 / \lambda 6731$ emission-line intensity ratio using the PyNeB routine (Luridiana et al. 2015), assuming the $t_{3}$ values obtained for each spaxel. For spaxels with no measurements of $t_{3}$, we use the mean $t_{3}$ value for each galaxy.

\section{RESULTS \& DISCUSSION}

Figures 1, 2, and 3 show emission-line flux distributions, flux line ratios, electron density, and electron temperature maps for Mrk 79, Mrk 348, and Mrk 607, respectively. The emission line ratios are corrected for dust extinction, as estimated from the $\mathrm{H} \alpha$ and $\mathrm{H} \beta$ fluxes (see Suppl. Mat.). The flux distributions for [O $\left.{ }_{\text {III }}\right] \lambda 4363$, [O $\left.{ }_{\text {III }}\right] \lambda 5007,\left[\mathrm{~N}_{\text {II }}\right] \lambda 5755$, and $\mathrm{H} \alpha$ emission lines are shown in the first row of each figure. There is no detection of the $\left[\mathrm{N}_{\text {II }}\right] \lambda 5755$ emission line in the spectra of Mrk 79 and thus, we do not show its flux map for this galaxy. The $N_{\mathrm{e}}$ maps present values in the range $\sim 100-2000 \mathrm{~cm}^{-3}$, with the highest values seen at the nucleus for all galaxies. The $[\mathrm{Ar}$ Iv] $\lambda 4711, \lambda 4740$ emission lines trace denser gas phases than the $[\mathrm{S}$ II] lines ratio. The [Ar IV] emission is not spatially resolved in our sample, but we measure the $[\mathrm{Ar}$ Iv] $\lambda 4711 / \lambda 4740$ line ratio by integrating the spectra within an $1^{\prime \prime} \times 1^{\prime \prime}$ centred at the nucleus. We obtain ratios of $0.64 \pm 0.20,0.44 \pm 0.15$ and $0.25 \pm 0.20$, which correspond to densities of $\sim 15200,31700$ and $80800 \mathrm{~cm}^{-3}$ for Mrk 79, Mrk 348 and Mrk 607, respectively.

The $\left[\mathrm{O}_{\text {III }}\right] \lambda 4363$ and $\left[\mathrm{N}_{\text {II }}\right] \lambda 5755$ flux distributions are spatially resolved (see Suppl. Mat.) in our sample. Thus, we use the fluxes of these lines to compute spatially resolved maps for $R_{\mathrm{O} 3}$ and $R_{\mathrm{N} 2}$. The [S $\left.\mathrm{II}\right]$ emission spreads over most of the GMOS field of view for all galaxies, allowing us to construct $R_{S 2}$ maps. We show these maps in Figs. 1, 2 and 3.

The bottom panels of Figs. 1, 2, and 3 show the $N_{\mathrm{e}}$ and $T_{\mathrm{e}}$ maps for Mrk 79, Mrk 348, and Mrk 607, respectively. These properties were computed from the $R_{S 2}, R_{\mathrm{O} 3}, R_{N 2}$ following the procedure described in Sec. 2. In all galaxies, we find the highest values of $R_{\mathrm{O} 3}$ along the AGN photo-ionization structure traced by the [O III] $\lambda 5007$ emission (Schmitt et al. 2003). Otherwise, smaller $R_{\mathrm{O} 3}$ values are derived mostly away from the AGN ionization axis, indicating higher temperatures at these locations. This indicates that, besides AGN photoionization, an additional process may be producing the observed emission. There are some differences in the $T_{\mathrm{e}}$ distributions observed in the three objects. For Mrk 348 and Mrk 79 very high temperature values ( $230000 \mathrm{~K})$ are observed in several spaxels. Most of the ionized gas emission in the inner $\mathrm{kpc}$ of these galaxies is produced in outflows with velocities of up to $200 \mathrm{~km} \mathrm{~s}^{-1}$ (Freitas et al. 2018; Riffel et al. 2013). The high temperature values are likely due to shocks produced by the

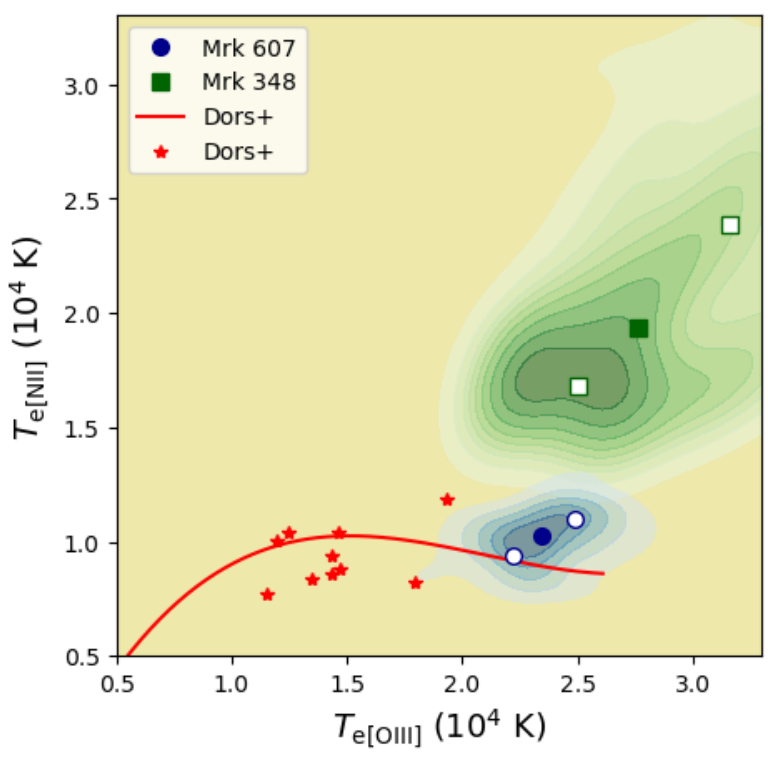

Figure 4. $T_{\mathrm{e}[\mathrm{OIII}]}$ vs. $T_{\mathrm{e}[\mathrm{NII}]}$. The green and blue density curves are for Mrk 348 and Mrk 607 respectively. The open squares and open circles show the 25th and 75th percentile $T_{\mathrm{e}}$ values for Mrk 348 and Mrk 607, respectively. The filled squares and circles show the median $T_{\mathrm{e}}$ values. The red stars are the AGN compilation of direct estimates of temperatures from Dors et al. (2017, 2020) and the red curve corresponds to predictions of their AGN photoionization models.

outflows, hence the gas reaches a maximum temperature in the immediate postshock region $\left(T_{\mathrm{e}} \propto V_{\mathrm{S}}^{2}\right.$, Contini 2019, where $V_{\mathrm{S}}$ is the shock velocity). For Mrk 79, it was possible to estimate $T_{\mathrm{e}}$ in the outskirt regions, where relatively low values $(10000-12000$ $\mathrm{K})$ are derived, which indicate a large temperature gradient in this object. For Mrk 348, the $T_{\mathrm{e}}$ maps show an increase in temperature from the nucleus to locations farther away from it, with $T_{\mathrm{e}}[\mathrm{NII}]$ increasing from 8000 to $\gtrsim 30000 \mathrm{~K}$. The $T_{\mathrm{e}[\mathrm{OIII}]}$ map shows values larger than $\sim 20000 \tilde{\mathrm{K}}$ in most locations, with slightly higher values seen in regions away from the nucleus. The $T_{\mathrm{e}}$ distributions found for Mrk 79 and Mrk 348 are distinct from the findings of Revalski et al. (2018) for Mrk 573 based on long slit data, where they find no systematic variations in temperature, with a mean value of $13500 \pm 650 \mathrm{~K}$. The $T_{\mathrm{e}[\mathrm{OIII}]}$ and $T_{\mathrm{e}[\mathrm{NII}]}$ maps for Mrk 607 show only small variations in temperature, with $T_{\mathrm{e}[\mathrm{OIII}]} \sim 20000 \mathrm{~K}$ and $T_{\mathrm{e}[\mathrm{NII}]} \sim 10000 \mathrm{~K}$. No clear evidence of outflows is seen in this galaxy (Freitas et al. 2018).

Figure 4 shows the $T_{\mathrm{e}[\mathrm{NII}]}$ versus $T_{\mathrm{e}[\mathrm{OIII}]}$ plot with the observed values for Mrk 607 and Mrk 348, which are compared to predictions of AGN photoionization models built with the CLOUDY code (Ferland et al. 2013) by Dors et al. (2020) and to integrated measurements for Seyfert 2 galaxies. While the points for Mrk 607 are seen in the same region occupied by the model predictions and by the integrated spectra, the points for Mrk 348 are located in a very distinct position, towards higher temperatures. This result, together with the fact that the [O III] emission in the nuclear region of this galaxy is mainly due to outflowing gas (Freitas et al. 2018), is a strong indication that outflows have an important effect on temperature structure of AGN hosts. We find that the temperature increases with [O I $] 6300 / \mathrm{H} \alpha$, a known tracer of shocks (e.g. Allen et al. 2008; Rich et al. 2014) and with the width of [O III]5007 (see Suppl. Mat.), indicating that shocks from AGN winds produce the high temperatures observed in Mrk 79 and Mrk 348. 
The spaxels which show the largest values of $T_{\mathrm{e}}$ in our sample are seen approximately perpendicular to the AGN ionization axis (clearly observed in Mrk 79, Fig. 1), indicating that shocks have a larger relative contribution to the line emission at these locations. An increase in the $\left[\mathrm{O}_{\mathrm{III}}\right]$ line width is seen at these locations (Sup. Mat.; Freitas et al. 2018), indicating the outflows are interacting with gas clouds, partially ionizing them and producing the observed line emission. Photo-ionized gas is a more efficient emitter of [O III] lines than shock-ionized gas, therefore, a possible shock contribution to the $\left[\mathrm{O}_{\mathrm{III}}\right]$ emission within the AGN ionization structure is overshadowed by the photoionization contribution. This result indicates that the outflows from Seyfert nuclei may have wide-opening angles as observed in luminous AGN (e.g. Kakkad et al. 2020) and predicted by theoretical models (Wagner et al. 2012; Ishibashi et al. 2019). Similar outflows in the equatorial plane of the torus have been reported in a few Seyfert galaxies (Riffel et al. 2014; Lena et al. 2015). The bipolar outflows observed in some cases (e.g. Crenshaw et al. 2010; Storchi-Bergmann et al. 2010) may be tracing only the photoionized part of the outflowing gas, as shocks are not efficient in the line production, when competing with photoionization (Zakamska \& Greene 2014), and thus the outflows are observed within the AGN ionization structure.

\section{CONCLUSIONS}

We present spatially resolved maps of the electron temperature within the inner few hundred parsecs of the Seyfert galaxies Mrk 79, Mrk 348, and Mrk 607, obtained from integral field spectroscopy. Our results indicate that shocks play an important role in the observed electron temperature distributions, as they can not be reproduced by AGN photoionization models only. This provides a caveat to studies of chemical abundances based on the usual methods for objects with shock/outflows signatures. Not taking this properly into account could yield biased estimates of the gas properties because shocks can produce an electron energy distribution distinct from the Maxwell-Boltzmann distribution. Shocks are more important in regions away from the AGN ionization axis, where they can be easily observed as the AGN radiation field is shielded by the nuclear dusty torus, while within the AGN ionization structure, photoionization is more efficient in producing line emission.

\section{ACKNOWLEDGEMENTS}

We thank to an anonymous referee for the valuable comments that helped us to improve the paper. This study was financed in part by Conselho Nacional de Desenvolvimento Científico e Tecnológico (202582/2018-3, 304927/2017-1 and 400352/2016-8), Fundação de Amparo à pesquisa do Estado do Rio Grande do Sul (17/2551-0001144-9 and 16/2551-0000251-7) and do Estado de São Paulo. AF acknowledges the support from grant PRIN MIUR201720173ML3WW_001. EPM acknowledges financial support from the project "Estallidos6" AYA2016-79724-C4. Based on observations obtained at the Gemini Observatory, which is operated by the Association of Universities for Research in Astronomy, Inc., under a cooperative agreement with the NSF on behalf of the Gemini partnership: the National Science Foundation (United States), National Research Council (Canada), CONICYT (Chile), Ministerio de Ciencia, Tecnología e Innovación Productiva (Argentina), Ministério da Ciência, Tecnologia e Inovação (Brazil), and Korea Astronomy and Space Science Institute (Republic of Korea).

\section{DATA AVAILABILITY}

The data used in this paper is available in the Gemini Science Archive under the project code GN-2014B-Q-87.

\section{REFERENCES}

Allen M. G., Groves B. A., Dopita M. A., Sutherland R. S., Kewley L. J., 2008, ApJS, 178, 20

Allington-Smith J., et al., 2002, PASP, 114, 892

Baldwin J. A., Phillips M. M., Terlevich R., 1981, PASP, 93, 5

Cardelli J. A., Clayton G. C., Mathis J. S., 1989, ApJ, 345, 245

Castro C. S., Dors O. L., Cardaci M. V., Hägele G. F., 2017, MNRAS, 467, 1507

Contini M., 2019, MNRAS, 488, 4487

Contini M., Viegas S. M., 2001, ApJS, 137, 75

Crenshaw D. M., Kraemer S. B., Schmitt H. R., Jaffé Y. L., Deo R. P., Collins N. R., Fischer T. C., 2010, AJ, 139, 871

D’Agostino J. J., et al., 2019, MNRAS, 487, 4153

Dahmer-Hahn L. G., et al., 2019, MNRAS, 489, 5653

Davies R. L., Kewley L. J., Ho I. T., Dopita M. A., 2014, MNRAS, 444, 3961

Dors O. L. J., Arellano-Córdova K. Z., Cardaci M. V., Hägele G. F., 2017, MNRAS, 468, L113

Dors O. L., Agarwal B., Hägele G. F., Cardaci M. V., Rydberg C.-E., Riffel R. A., Oliveira A. S., Krabbe A. C., 2018, MNRAS, 479, 2294

Dors O. L., Monteiro A. F., Cardaci M. V., Hägele G. F., Krabbe A. C., 2019, MNRAS, 486, 5853

Dors O. L., Maiolino R., Cardaci M. V., Hägele G. F., Krabbe A. C., PérezMontero E., Armah M., 2020, MNRAS, 496, 3209

Feltre A., Charlot S., Gutkin J., 2016, MNRAS, 456, 3354

Ferland G. J., Netzer H., 1983, ApJ, 264, 105

Ferland G. J., et al., 2013, Rev. Mex. Astron. Astrofis., 49, 137

Freitas I. C., et al., 2018, MNRAS, 476, 2760

Guo Y., et al., 2020, ApJ, 898, 26

Hägele G. F., Díaz Á. I., Terlevich E., Terlevich R., Pérez-Montero E., Cardaci M. V., 2008, MNRAS, 383, 209

Ishibashi W., Fabian A. C., Reynolds C. S., 2019, MNRAS, 486, 2210

Kakkad D., et al., 2020, A\&A, 642, A147

Kewley L. J., Dopita M. A., Sutherland R. S., Heisler C. A., Trevena J., 2001, ApJ, 556, 121

Lena D., et al., 2015, ApJ, 806, 84

Luridiana V., Morisset C., Shaw R. A., 2015, A\&A, 573, A42

Marchese E., Della Ceca R., Caccianiga A., Severgnini P., Corral A., Fanali R., 2012, A\&A, 539, A48

Mignoli M., et al., 2019, A\&A, 626, A9

Miller B. P., Brandt W. N., Schneider D. P., Gibson R. R., Steffen A. T., Wu J., 2011, ApJ, 726, 20

Nagao T., Murayama T., Taniguchi Y., 2001, ApJ, 549, 155

Nagar N. M., Wilson A. S., Mulchaey J. S., Gallimore J. F., 1999, ApJS, 120, 209

Osterbrock D. E., Ferland G. J., 2006, Astrophysics of gaseous nebulae and active galactic nuclei

Pérez-Montero E., Dors O. L., Vílchez J. M., García-Benito R., Cardaci M. V., Hägele G. F., 2019, MNRAS, 489, 2652

Revalski M., Crenshaw D. M., Kraemer S. B., Fischer T. C., Schmitt H. R., Machuca C., 2018, ApJ, 856, 46

Rich J. A., Kewley L. J., Dopita M. A., 2011, ApJ, 734, 87

Rich J. A., Kewley L. J., Dopita M. A., 2014, ApJ, 781, L12

Riffel R. A., Storchi-Bergmann T., Winge C., 2013, MNRAS, 430, 2249

Riffel R. A., Storchi-Bergmann T., Riffel R., 2014, ApJ, 780, L24

Ruschel-Dutra D., 2020, danielrd6/ifscube v1.0, doi:10.5281/zenodo.3945237, https://doi.org/10.5281/ zenodo. 3945237

Schmitt H. R., Ulvestad J. S., Antonucci R. R. J., Kinney A. L., 2001, ApJS, 132, 199 
Schmitt H. R., Donley J. L., Antonucci R. R. J., Hutchings J. B., Kinney A. L., 2003, ApJS, 148, 327

Stasińska G., 1984, A\&A, 135, 341

Storchi-Bergmann T., Schmitt H. R., Calzetti D., Kinney A. L., 1998, AJ, 115,909

Storchi-Bergmann T., Lopes R. D. S., McGregor P. J., Riffel R. A., Beck T., Martini P., 2010, MNRAS, 402, 819

Wagner A. Y., Bicknell G. V., Umemura M., 2012, ApJ, 757, 136

Wylezalek D., Flores A. M., Zakamska N. L., Greene J. E., Riffel R. A., 2020, MNRAS, 492, 4680

Zakamska N. L., Greene J. E., 2014, MNRAS, 442, 784

\section{APPENDIX A: EXAMPLES OF FITS OF THE EMISSION-LINE PROFILES}

The fitting of the spectra was performed using the IFSCUBE code (Ruschel-Dutra 2020), as mentioned in Section 2. We perform a simultaneous fit of the following emission lines: $\mathrm{H} \gamma$, [O III] $\lambda$ 4363, He II $\lambda$ 4686, H $\beta$, [O III] $\lambda \lambda$ 4959,5007, [Fe viI] $\lambda$ 5721, $\left[\mathrm{N}_{\mathrm{II}}\right] \lambda$ 5755, [O I] $\lambda \lambda$ 6300,6364, [N $\left.\mathrm{N}_{\mathrm{II}}\right] \lambda \lambda$ 6548,6583, $\mathrm{H} \alpha$, and $\left[\mathrm{S}_{\text {II }}\right] \lambda \lambda 6716,6731$. We allow the fit of up to three Gaussian components per emission line, tying the kinematics of the components of emission lines from the same parent ion, and keeping fixed the $\left[\mathrm{N}_{\text {II }}\right] \lambda 6583 / \lambda 6548$ and $\left[\mathrm{O}_{\text {III }}\right] \lambda 5007 / \lambda 4959$ flux ratios to their theoretical values of 3.06 and 2.98 , respectively. We provide additional Gaussian components to account for the broad components of the $\mathrm{H}$ recombination lines in Mrk 79, which hosts a type 1 AGN. We fit the continuum emission by a fifth order polynomial. The code fits the nuclear spaxel using initial guesses provided by the user and than follows a spiral pattern from the nucleus outwards, using optimized guesses from the best-fit parameters from neighboring spaxels at distances smaller than 0 .' 35 , by using the refit parameter of the IFSCUBE code. If the line profile is well reproduced by less than three Gaussian components at a specific spaxel, the amplitude of needless components are set to zero and the code returns the bestfit parameters of the remaining components. We do not associate a physical meaning with each separate Gaussian component. The fluxes of each emission line is obtained by the sum of the fluxes of the individual components. The median flux uncertainties are $12 \%$ for $\left[\mathrm{O}_{\mathrm{III}}\right] \lambda 4363$, and $15 \%$ for $[\mathrm{N}$ II] $\lambda 5755$ but smaller than $5 \%$ for the strong lines (e.g. [O $\mathrm{III}] \lambda 5007)$.

Figure A1 shows examples of the fits of the strong emission lines: $\mathrm{H} \beta,\left[\mathrm{O}_{\text {III }}\right] \lambda \lambda$ 4959,5007, $\mathrm{H} \alpha$ and [N $\left.\mathrm{II}\right] \lambda \lambda$ 6548,6583. Figure A2 shows examples of the Gaussian fits of the $\mathrm{H} \gamma+\left[\mathrm{O}_{\text {III }}\right] \lambda 4363$ (top) and [ $\left.\mathrm{N}_{\mathrm{II}}\right] \lambda 5755$ (bottom) emission lines. The emission lines are well reproduced by the Gaussian models and the $\mathrm{H} \gamma$ and [O III] $] 4363$ are clearly separated.

\section{APPENDIX B: CURVES-OF-GROWTH FOR THE [O III] AND [N II] EMISSION}

The [O $\mathrm{III}] \lambda 4363$ and $\left[\mathrm{N}_{\mathrm{II}}\right] \lambda 5755$ flux distributions extend to distances larger than the FWHM of the point spread function (PSF) in all galaxies. However, the emission-line flux distributions of an unresolved nuclear source can be smeared by the seeing to distances larger than the FWHM of the PSF. To further check if the $\left[\mathrm{O}_{\mathrm{III}}\right] \lambda 4363$ and $\left[\mathrm{N}_{\mathrm{II}}\right] \lambda 5755$ flux distributions in our sample are spatially resolved, we follow Kakkad et al. (2020) and compute their curves-of-growth (COGs). We compare the emission-line and PSF COGs, with the later obtained from images of field stars in the GMOS acquisition images for Mrk 348 and Mrk 607, and from the
$\mathrm{H} \alpha$ emission from the broad line region of Mrk 79. First, we obtain the integrated flux within a circular aperture of 0 '. $^{\prime} 15$ centred at the nucleus, normalize the emission-line flux distribution by this value and then compute the integrated fluxes increasing the radius in steps of 0.' 15. In Fig. B1 we show the corresponding COGs, which show the $\left[\mathrm{O}_{\mathrm{III}}\right] \lambda 4363$ emission presents an excess in flux as compared to the PSF, indicating that the $\left[\mathrm{O}_{\mathrm{III}}\right] \lambda 4363$ emission is spatially resolved in all galaxies. The $[\mathrm{N}$ II $] \lambda 5755$ emission in Mrk 348 is also spatially resolved, while in Mrk 607 it is marginally resolved.

\section{APPENDIX C: EMISSION-LINE RATIO MAPS}

The gas reddening is obtained from the $\mathrm{H}_{\alpha}$ and $\mathrm{H}_{\beta}$ observed fluxes by adopting the theoretical line ratio of $I_{H \alpha} / I_{H \beta}=2.86$ for Case B H I recombination, an electron temperature of $T_{\mathrm{e}}=10000 \mathrm{~K}$ (Osterbrock \& Ferland 2006) and $R_{V}=3.1$. Using the coefficients from the extinction law of Cardelli et al. (1989) the colour excess is given by:

$E(B-V)=2.32 \log \left(\frac{\left(F_{\mathrm{H} \alpha} / F_{\mathrm{H} \beta}\right)}{2.86}\right)$,

where $F_{\mathrm{H} \alpha}$ and $F_{\mathrm{H} \beta}$ are the observed fluxes of the $\mathrm{H} \alpha$ and $\mathrm{H} \beta$ emission lines at each spaxel.

Figures $\mathrm{C} 1, \mathrm{C} 2$ and $\mathrm{C} 3$ show the $E(B-V)$ maps for Mrk 79, Mrk 348 and Mrk607, respectively. These figures also show the $\left[\mathrm{O}_{\mathrm{III}}\right] 5007 / \mathrm{H} \beta,\left[\mathrm{O}_{\mathrm{I}}\right] 6300 / \mathrm{H} \alpha,\left[\mathrm{N}_{\mathrm{II}}\right] 6583 / \mathrm{H} \alpha$ and $\left(\left[\mathrm{S}_{\text {II }}\right] 6716+6731\right) / \mathrm{H} \alpha$ flux ratio maps and $W_{80}$ maps for the [O III]5007 emission line. The flux ratio maps are corrected by extinction using the $E(B-V)$ values and adopting the extinction law by Cardelli et al. (1989). The $W_{80}$ parameter measures the width of the emission-line profile that includes $80 \%$ of the integrated line flux. Values larger than $500-600 \mathrm{~km} \mathrm{~s}^{-1}$ are commonly attributed to ionized gas outflows (Zakamska \& Greene 2014; Kakkad et al. 2020; Wylezalek et al. 2020).

The flux-line ratios for all galaxies are typical of Seyfert nuclei, as already shown in Freitas et al. (2018), based on the fitting of the emission-line profiles by single Gaussian curves and using the same data considered here. Fig. C4 shows the [O I]-BPT diagram (Baldwin et al. 1981) for the spaxels with $T_{\mathrm{e}[\mathrm{OIII}]}$ estimates. The points are color coded using by the $T_{\mathrm{e}}$ [OIII] values of each spaxel, as shown in the color bar.

The $\left[\mathrm{O}_{\mathrm{I}}\right] \lambda 6300 / \mathrm{H} \alpha$ line ratio is a tracer of shocks in neutral gas (e.g., Allen et al. 2008; Rich et al. 2011, 2014). If the velocity dispersion of $\left[\mathrm{O}_{\mathrm{I}}\right.$ ] is larger than $150 \mathrm{~km} \mathrm{~s}^{-1}$ and $\left[\mathrm{O}_{\mathrm{I}}\right] \lambda 6300 / \mathrm{H} \alpha \gtrsim$ 0.1 , shocks with velocities in the range of $160-300 \mathrm{~km} \mathrm{~s}^{-1}$ are the dominant excitation mechanism of the $\left[\mathrm{O}_{\mathrm{I}}\right]$ emission line. The upper right panels of Figs. C1, C2 and C3 show a plot of [O I] $\lambda 6300 / \mathrm{H} \alpha$ vs. $T_{\mathrm{e}[\mathrm{OIII}]}$ for the galaxies of our sample. For Mrk 79 and Mrk 348, the mean $\left[\mathrm{O}_{\mathrm{I}}\right] \lambda 6300 / \mathrm{H} \alpha$ ratio increases with increasing temperature, reaching individual ratio values of up to 0.25 and 0.50 for Mrk 79 and Mrk 348. Mrk 607 presents overall smaller values of [O I] $] 6300 / \mathrm{H} \alpha$ and there is no trend of this ratio with the electron temperature. This result indicates that shocks play an important role in the production of the highest electron temperatures in Mrk 79 and Mrk 348. The observed [O I] $\lambda 6300 / \mathrm{H} \alpha$ in Mrk 79 and Mrk 348 can be produced by shocks with velocities of $V_{S} \gtrsim 300 \mathrm{~km} \mathrm{~s}^{-1}$ (Allen et al. 2008), which are smaller than the observed outflow velocities traced by the $W_{80}$ parameter.

The shocks in Mrk 79 and Mrk 348 may be produced by outflows. Both galaxies show well defined bipolar outflows along the 

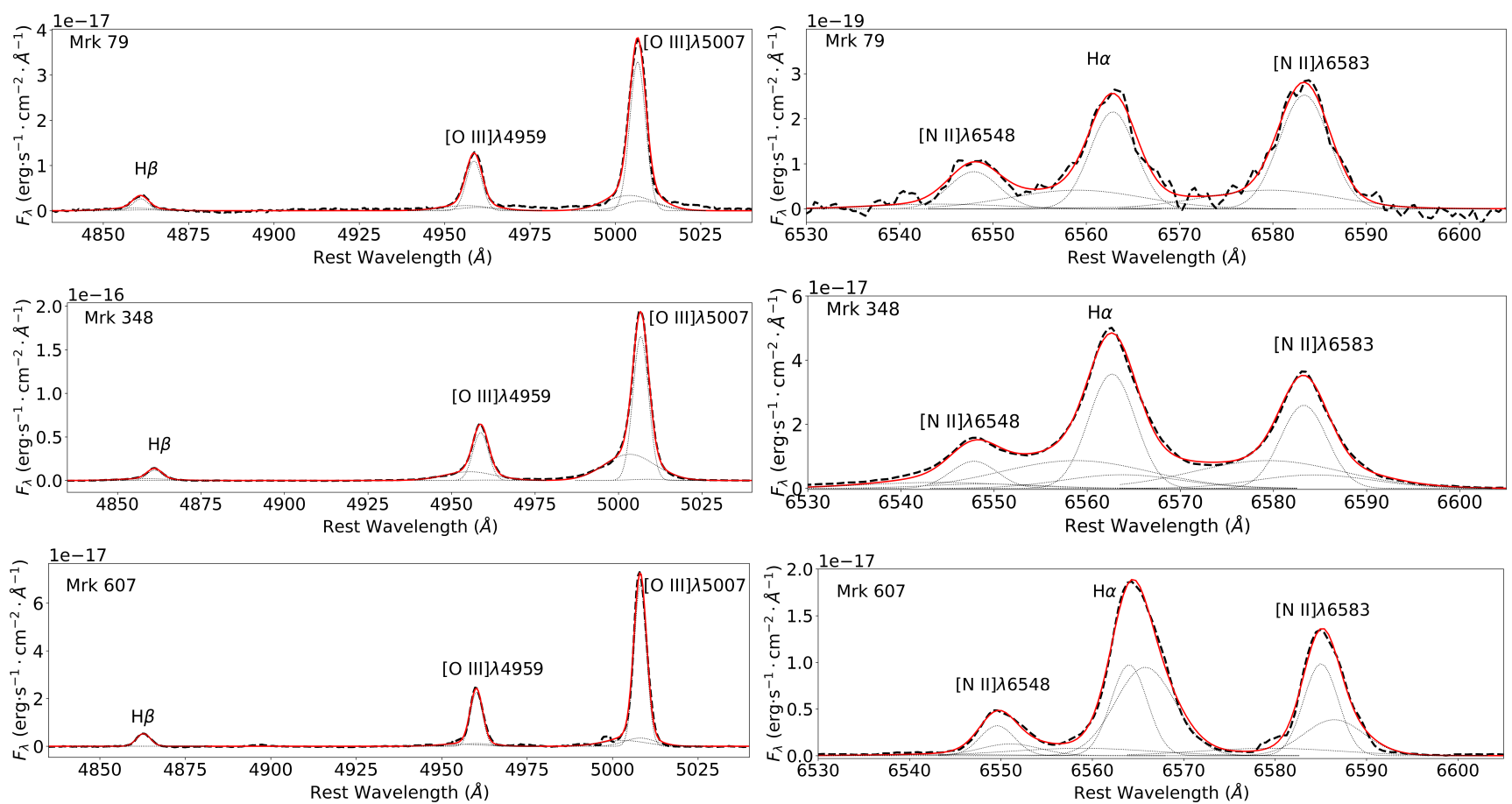

Figure A1. Examples of $\left[\mathrm{O}_{\mathrm{III}}\right]+\mathrm{H} \beta$ (left) and $\left[\mathrm{N}_{\mathrm{II}}\right]+\mathrm{H} \alpha$ (right) emission-line profiles for Mrk 79 (top), Mrk 348 (middle) and Mrk 607 (bottom). The continuum-subtracted spectrum is shown in dashed black lines, the model in continuous red and the individual components are shown as dotted lines. For Mrk 79, the contribution of the broad $\mathrm{H} \beta$ and $\mathrm{H} \alpha$ emission has also been subtracted.
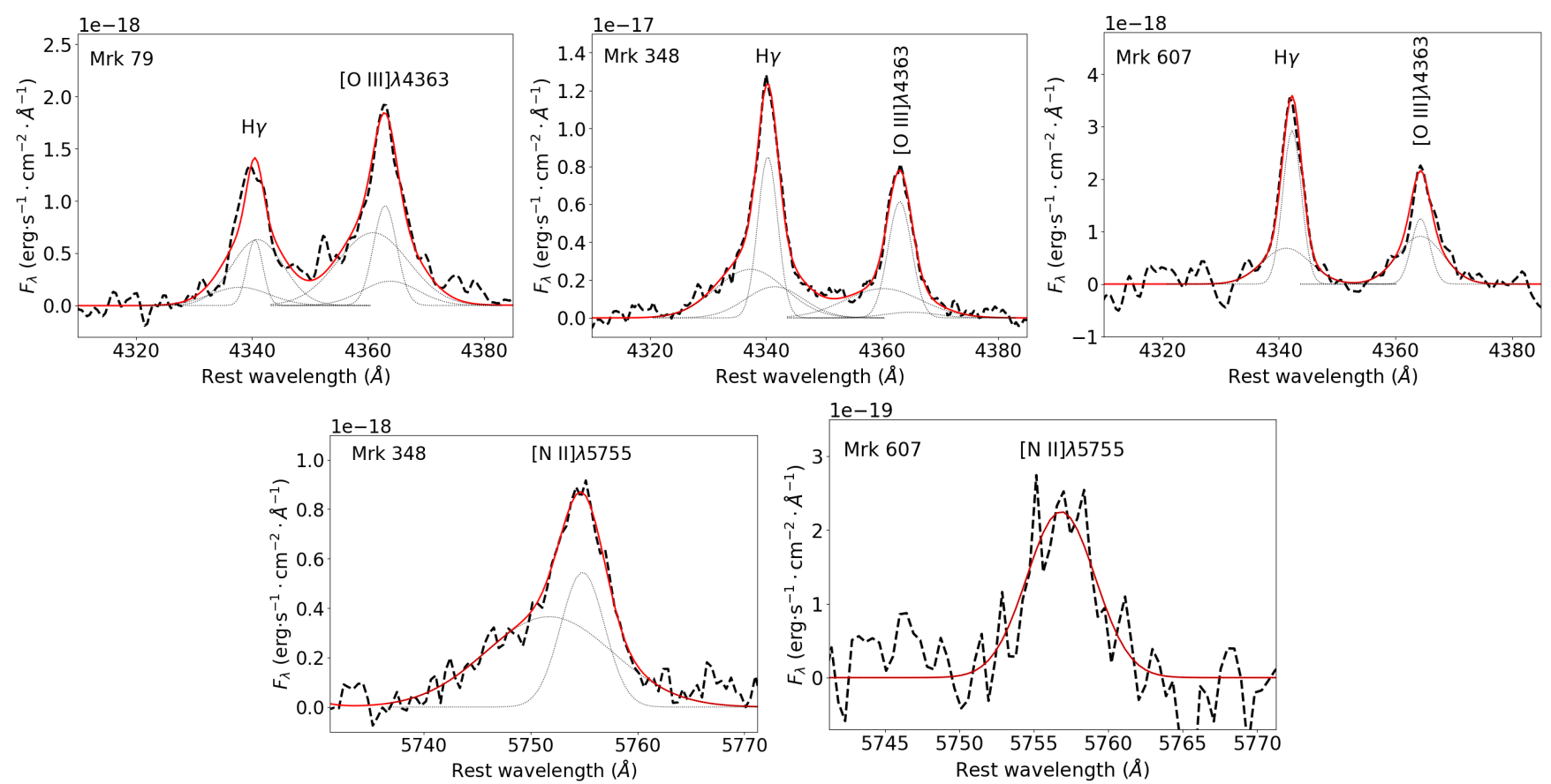

Figure A2. Examples of the Gaussian fits of the $\mathrm{H} \gamma+\left[\mathrm{O}_{\mathrm{III}}\right] \lambda 4363$ (top) and [N $\left.\mathrm{II}\right] \lambda 5755$ (bottom). The continuum-subtracted spectrum is shown in dashed black lines, the model in continuous red and the individual components are shown as dotted lines. For Mrk 79, the contribution of the broad $\mathrm{H} \gamma$ emission has also been subtracted. 

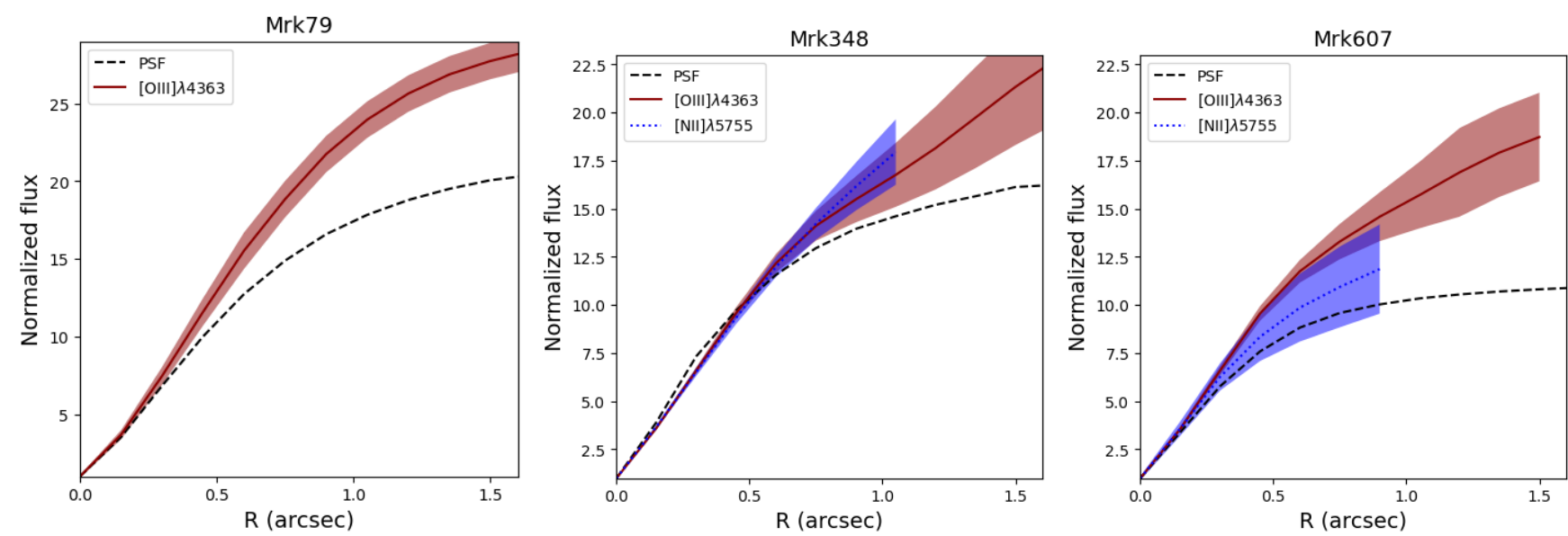

Figure B1. Curves-of-growth for Mrk 79 (left), Mrk 348 (center) and Mrk 607 (right). The black dashed line corresponds to the PSF as obtained from the flux distribution of the broad component of $\mathrm{H} \alpha$ for Mrk 79 and from field stars on the acquisition images of Mrk 348 and Mrk 607. The red and blue lines show the $\left[\mathrm{O}_{\text {III }}\right] \lambda 4363$ and $\left[\mathrm{N}_{\text {II }}\right] \lambda 5755$ curve-of-growth, respectively. The shaded regions delineate the $1 \sigma$ flux uncertainties. The $\left[\mathrm{N}_{\text {II }}\right] \lambda 5755$ is not detected for Mrk 79. These curves were constructed by normalizing the flux distributions within the inner 0 "' 15 radius and computing the fluxes within apertures increasing in steps of 0 .' 15 each.

AGN ionization axis (Riffel et al. 2013; Freitas et al. 2018). In addition, the high $W_{80}$ values for the [O III] 5007 line in these galaxies (bottom-left panels of Figs. C1 and C2) indicate that the presence of outflows not only along the ionization axis, but also in locations away from it. Values of $W_{80} \gtrsim 500 \mathrm{~km} \mathrm{~s}^{-1}$ are commonly interpreted as tracers of AGN outflows (Zakamska \& Greene 2014; Kakkad et al. 2020; Wylezalek et al. 2020). For Mrk 79, the highest $W_{80}$ values are observed perpendicular to the AGN ionization axis, which is indicated as a continuous line in the $W_{80}$ map of Fig. C1. This galaxy shows radio emission approximately along the same direction of the AGN axis (Schmitt et al. 2001), while Mrk 348 and Mrk 607 do not present signature of radio jets (Nagar et al. 1999). The high $W_{80}$ values in regions away from the AGN ionization cone indicates that the AGN winds are more spherical and not restricted to the ionization cone, in agreement with observations of luminous AGNs (e.g. Kakkad et al. 2020) and predicted by theoretical models (Ishibashi et al. 2019). Similar outflows approximately in the equatorial plane of the torus have been observed in a few Seyfert galaxies using optical and near-IR IFS (Riffel et al. 2014; Lena et al. 2015). The interaction of these outflows with the ambient gas may produce shocks, responsible for the increase of the temperature in regions away from the AGN ionization cone.

This paper has been typeset from a $\mathrm{TE}_{\mathrm{E}} \mathrm{X} / \mathrm{LT} \mathrm{T} \mathrm{X}$ file prepared by the author. 

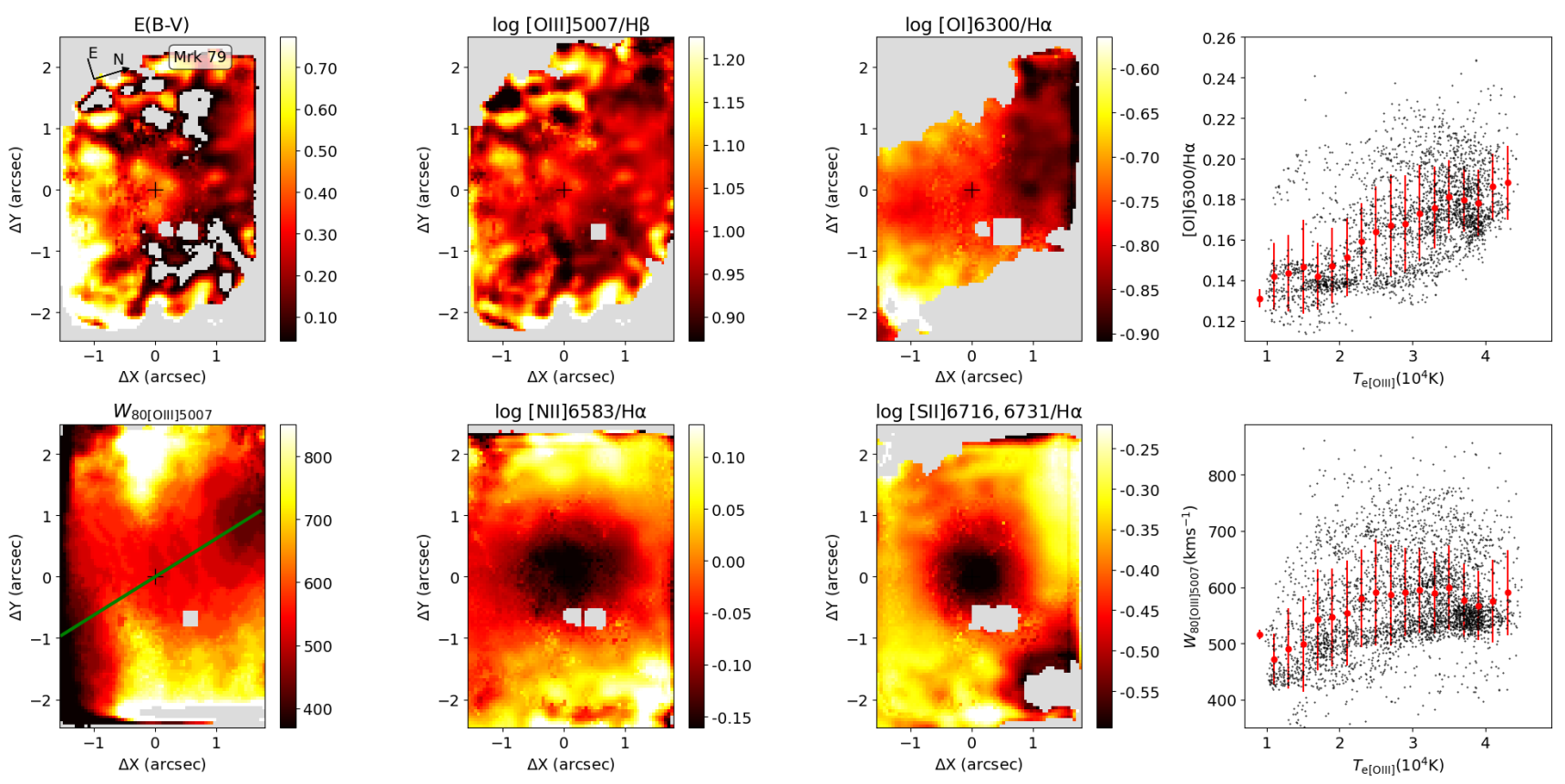

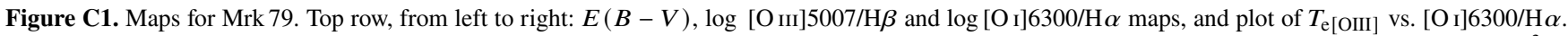
The black points show the observed $T_{\mathrm{e}[\mathrm{OIII}]}$ and $\left[\mathrm{O}_{\mathrm{I}}\right] / \mathrm{H} \alpha$ values at each spaxel and the red circles show the mean values within $T_{\mathrm{e}[\mathrm{OIII}]}$ bins of $2 \times 10^{3} \mathrm{~K}$ and the error bars are the standard deviations of the $\left[\mathrm{O}_{\mathrm{I}}\right] / \mathrm{H} \alpha$ within each bin. Bottom row, from left to right: $W_{80}$ map for the $\left[\mathrm{O}_{\text {III }}\right] 5007 \mathrm{emission}$ line - the

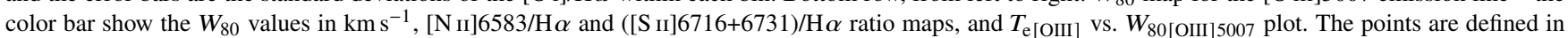
the same way as in the $T_{e}$ vs. [O I]/H $\alpha$ plot. The green line shows the AGN ionization axis, as measured by Schmitt et al. (2003) using Hubble Space Telescope $[\mathrm{O}$ III $] 5007$ narrow band images.
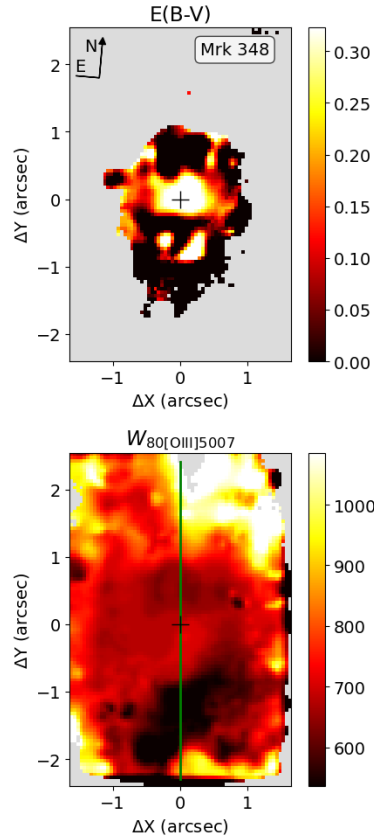
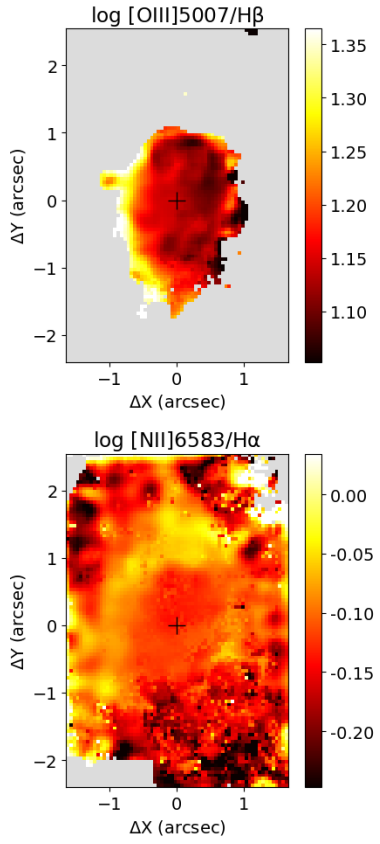
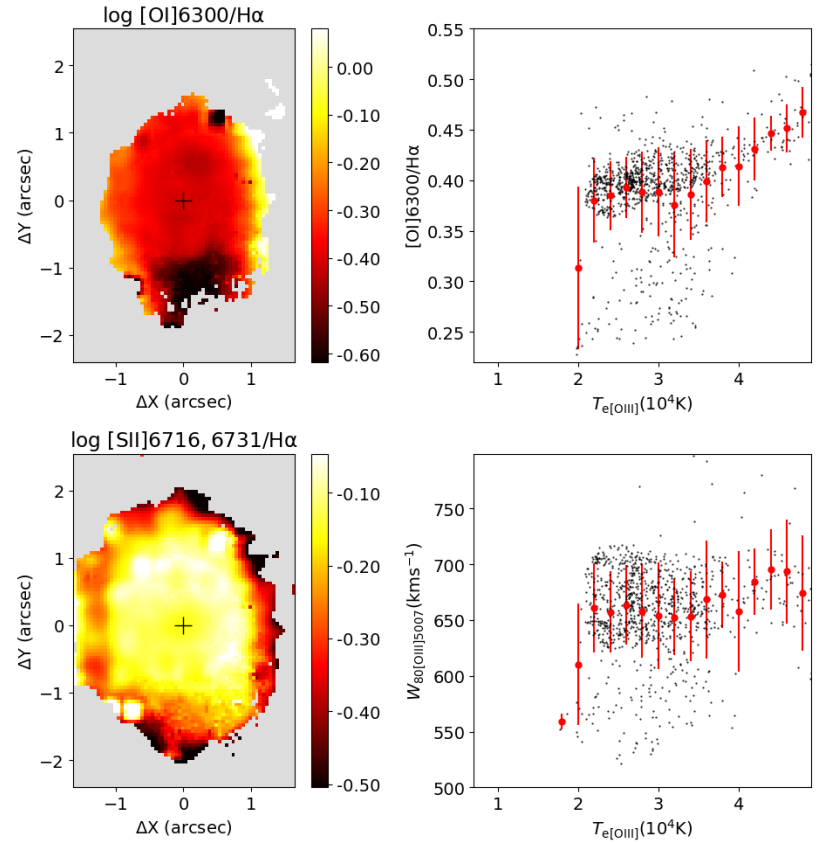

Figure C2. Same as Fig. C1, but for Mrk 348. 

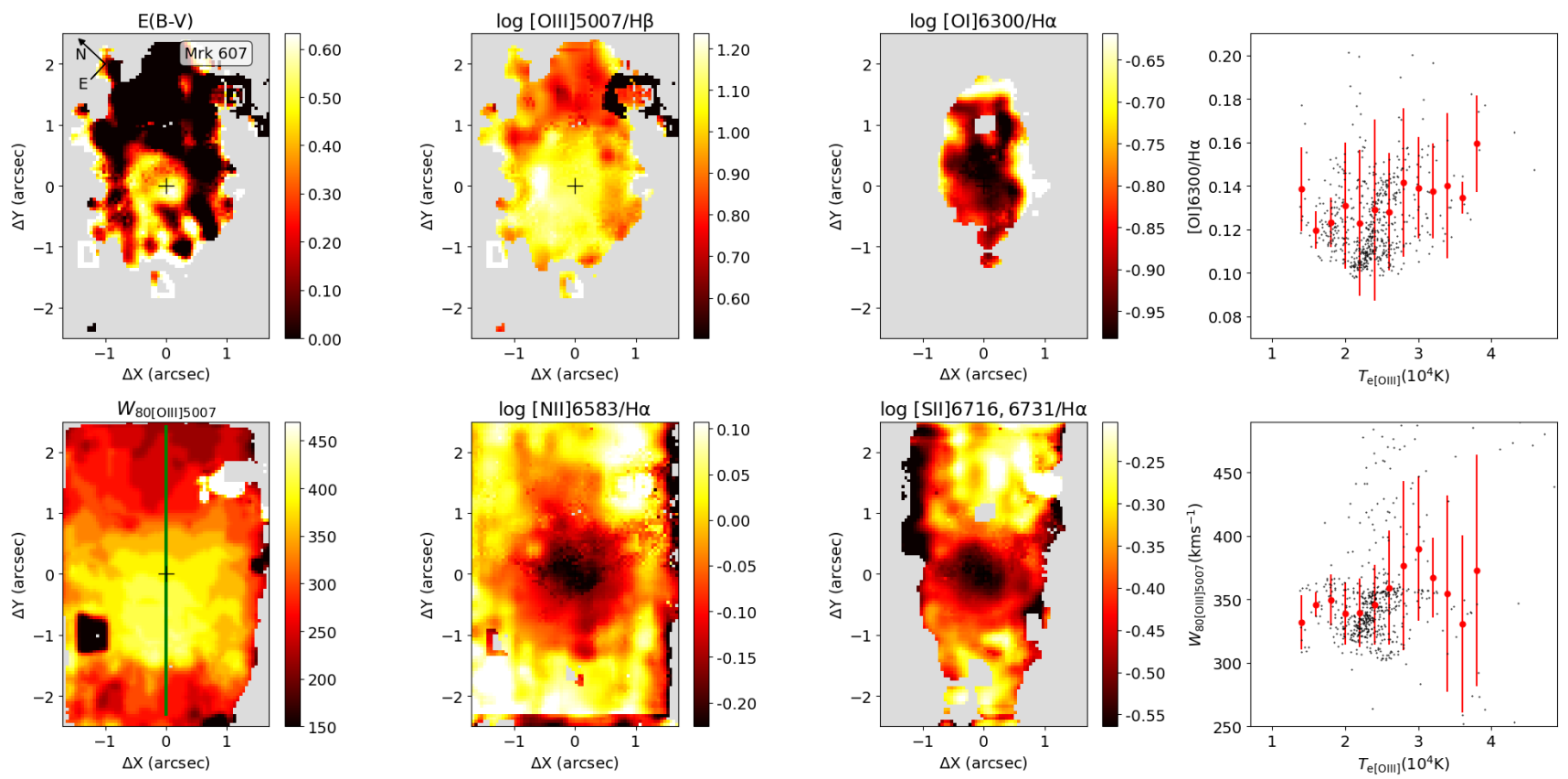

Figure C3. Same as Fig. C1, but for Mrk 607.
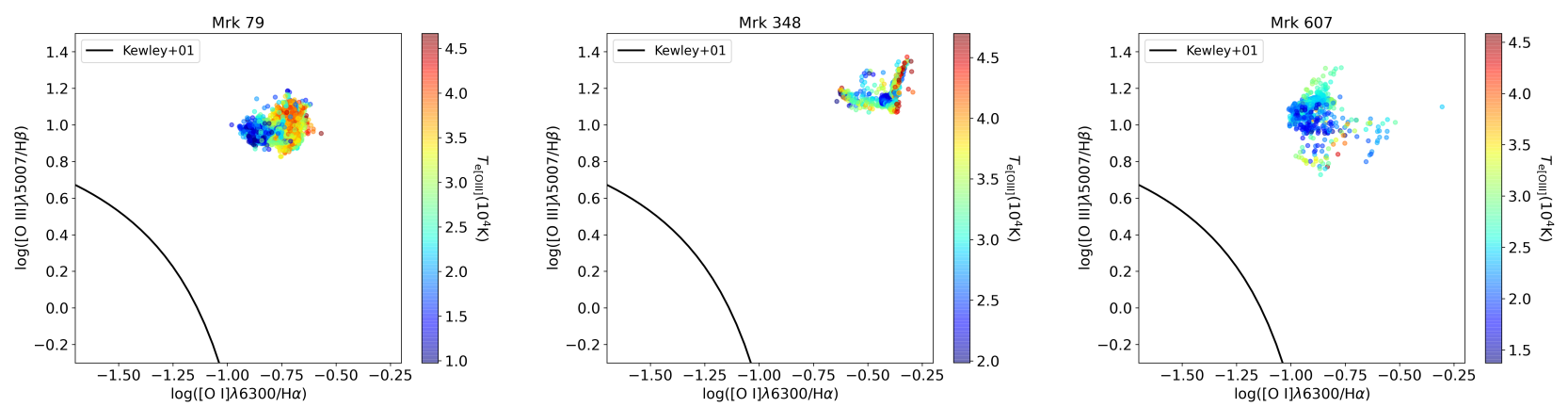

Figure C4. BPT [O I]-based diagrams for the spaxels with electron temperature measurements for Mrk 79 (left), Mrk 348 (middle) and Mrk 607 (right). The continuous line is from Kewley et al. (2001). 